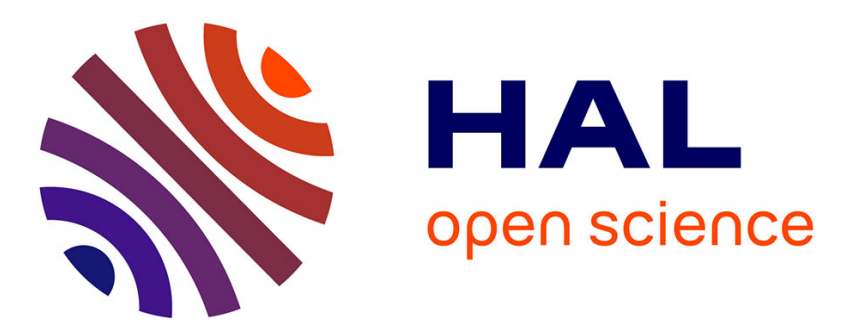

\title{
Influence of physical parameters and operating conditions for structural integrity of mechanical system subjected to squeal noise
}

\author{
Kevin Soobbarayen, Sébastien Besset, Jean-Jacques Sinou
}

\section{To cite this version:}

Kevin Soobbarayen, Sébastien Besset, Jean-Jacques Sinou. Influence of physical parameters and operating conditions for structural integrity of mechanical system subjected to squeal noise. Key Engineering Materials, 2013, 569-570, pp.1076-1084. 10.4028/www.scientific.net/KEM.569-570.1076 . hal-00914395

\section{HAL Id: hal-00914395 \\ https://hal.science/hal-00914395}

Submitted on 5 Dec 2013

HAL is a multi-disciplinary open access archive for the deposit and dissemination of scientific research documents, whether they are published or not. The documents may come from teaching and research institutions in France or abroad, or from public or private research centers.
L'archive ouverte pluridisciplinaire HAL, est destinée au dépôt et à la diffusion de documents scientifiques de niveau recherche, publiés ou non, émanant des établissements d'enseignement et de recherche français ou étrangers, des laboratoires publics ou privés. 


\title{
Influence of physical parameters and operating conditions for structural integrity of mechanical system subjected to squeal noise
}

\author{
K. Soobbarayen, S. Besset and J.-J. Sinou \\ Laboratoire de Tribologie et Dynamique des Systèmes, UMR CNRS 5513, \\ Ecole Centrale de Lyon, 36 avenue Guy de Collongue 69134 Ecully Cedex, France \\ \{kevin.soobbarayen, sebastien.besset, jean-jacques.sinou\}@ec-lyon.fr
}

Keywords: non-linear vibrations, acoustic radiation, brake squeal, friction

\begin{abstract}
This work proposes to study the effects of physical parameters and loading conditions on both dynamic and acoustic responses of a brake system subjected to squeal. A simplified brake system model composed of a disc and a pad is investigated. The friction interface is modeled by introducing linear and non-linear stiffnesses at several local nodes to model contact. The classical Coulomb law is applied to model friction and the friction coefficient is assumed to be constant. A stability analysis of this system is performed with respect to the friction coefficient and the hydraulic brake pressure. The system presents up to four instabilities and the focus is on a single instability case with a fundamental frequency of $930 \mathrm{~Hz}$. For this case, self-excited vibrations are investigated for two loading conditions: static and ramp loadings. Time responses for these cases are significantly different: the amplitude of the case with ramp loading is 130 times higher than the amplitude of the static load case. Spectrum analysis are performed by the Continuous Wavelet Transform and the response associated with the static load is composed of the fundamental frequency and its harmonic components. Time response with the progressive load is composed of two fundamental frequencies, their harmonic components and linear combinations which are not expected according to the stability analysis. Noise emissions for these two loading conditions present significantly different features in terms of level and directivity. It is noted that levels in the near field are about $127 \mathrm{~dB}$ for the static load and $193 \mathrm{~dB}$ for the progressive load. Moreover, the directivity patterns in the near and far fields are composed of lobes for the static load and circular wave front lines for the ramp loading.
\end{abstract}

\section{Introduction}

Disc brake systems are composed of two main components that are a circular disc and a pad. Over the interface between the disc and the pad, complex phenomena of contact and friction occur and these non-linearities can lead to self-excited vibrations induced by friction which produce squeal noise. For a complete background about brake technology and the problem of contact in automotive disc brake, one can refer to $[1,2]$. Squeal occurrences are still difficult to predict and many models which are capable of reproducing squeal events have been proposed. Mechanisms of friction-induced vibrations which lead to squeal are complex and references can be found in [3]. Studies that have been carried out are based on classical stability analysis of linearized equations of motion. In some cases, they are able to characterize squeal with respect to several parameters such as the friction coefficient, geometrical parameters, material properties or damping. However, stability analysis does not allow for the prediction of the amplitude of the stationary response or the frequencies which compose the spectrum. So, temporal integration is carried out to characterize cases which lead to squeal $[4,5]$. These non-linear time integrations are initialized with the quasi-static sliding equilibrium configuration which corresponds to the brake configuration under the hydraulic braking pressure. Considering a slight disturbance around the sliding equilibrium point, self-excited vibrations are generated. Generally, the main neglected phenomena are vibrations during the achievement of the sliding equilibrium point due to the evolution of the loading conditions $[4,5]$.

In this paper, we first focus on the influence of physical parameters, such as hydraulic pressure and friction coefficient, over the stability of a simplified brake system. Secondly, the influence of loading conditions over the non-linear dynamic response and noise emissions during squeal event is characterized: static and progressive loads cases are compared. 


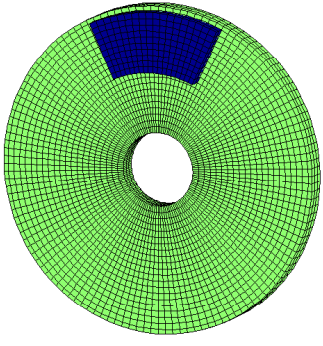

(a)

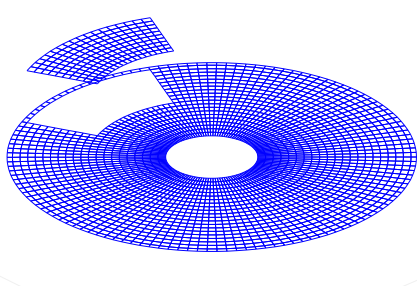

(b)

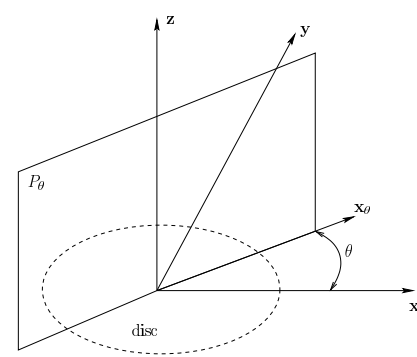

(c)

Fig. 1: Brake system model. (a): simplified brake system; (b): boundary element mesh; (c): observation plane $P_{\theta}$ used to display the acoustic intensity

\section{Brake system model and loading conditions}

Brake model. The brake system is modeled with a circular disc, where the inner radius is clamped, and a pad which can only translate along the normal direction (see Figure 1 (a)). There are about 34000 degrees of freedom and eight-node linear hexahedron elements are used. Moreover, a hydraulic pressure is applied over the back-plate of the pad.

In order to calculate the acoustic radiation, a boundary element model is used and it is composed of the upper surface of the disc without the friction interface and the upper surface of the pad (see Figure 1 (b)). The friction interface only radiates during loss of contact configurations so it can be assumed that this area does not contribute to the global acoustic radiation: it is not considered in the boundary element model. Moreover, the gap between the two previous surfaces contains fluid and allows acoustic interactions: for example the pad acoustic pressure can be reflected over the disc. To avoid this coupling, pressure fields over the disc and the pad meshes will be calculated separately. In free space, global radiations composed of both pad and disc noise emissions will be considered. For the acoustic study, parts of the Open BEM toolbox are used [6]. For more details about the boundary element method, one can refer to [7].

Contact/friction formulation. The friction interface is modeled by introducing nine uniformly spaced contact elements. Contact and loss of contact configurations are taken into account so that the disc and the pad can separate at several local nodes during the braking process. Moreover, the nonlinear contact force is described by a classical cubic law which fits experimental results as explained in [8]. During contact configuration, the normal contact force is of the form $F_{\text {contact }}=k_{L} \delta+k_{N L} \delta^{3}$ along the z-direction, where $\delta$ denotes the penetration between the disc and the pad, $k_{L}$ and $k_{N L}$ denote the linear and the cubic stiffnesses respectively. During loss of contact, $F_{\text {contact }}=0$.

The friction coefficient $\mu$ is assumed to be constant for the sake of simplicity and the classical Coulomb law is applied. Moreover, the rotation velocity of the disc is assumed to be such that the orthoradial velocity sign does not change during the braking process. So, the friction force is of the form $F_{\text {friction }}=\mu F_{\text {contact }}$ over the friction interface and along the direction of the orthoradial velocity.

Loading condition. The vector $\mathbf{F}(t)$ denotes the external force vector due to braking pressure. In the present study, two cases will be studied: the first one considers a static load $F_{\text {max }}$ by neglecting the ramp loading. The second one corresponds to a ramp loading defined by Equation 1.

$$
\mathbf{F}(t)= \begin{cases}\frac{t}{t_{R}} \mathbf{F}_{\max } & \text { if } t \leq t_{R} \\ \mathbf{F}_{\max } & \text { otherwise }\end{cases}
$$

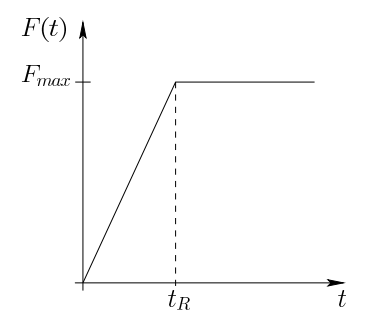


where $t_{R}$ is the duration of the linear part of $\mathbf{F}(t), t$ is the time and $\mathbf{F}_{\text {max }}$ is the force vector associated with the maximum brake pressure $P_{\max }$.

Equation of motion of the brake system. The Craig and Bampton method is applied to reduce the finite element model of the brake system under study. The first hundred eigenmodes of the complete system and all the attachment eigenmodes are retained. This reduction basis provides a good correlation between the complete and the reduced models up to $20 \mathrm{kHz}$. This frequency corresponds to the maximum audible frequency, so it allows for perform squeal noise calculation. Finally, the equations of motion for the reduced model are given by Equation 2:

$$
\mathbf{M} \ddot{\mathbf{X}}+\mathbf{C} \dot{\mathbf{X}}+\mathbf{K X}=\mathbf{F}_{\mathbf{N L}}(\mathbf{X})+\mathbf{F}(t)
$$

where $\mathbf{M}, \mathbf{C}$ and $\mathbf{K}$ are mass, damping and stiffness matrices, $\mathbf{X}$ is the generalized displacement vector and the dot denotes the derivative with respect to time. $\mathbf{F}_{\mathbf{N L}}(\mathbf{X})$ corresponds to the non-linear force vector which contains contact and friction force vectors. The damping matrix is computed by using a modal damping: we consider a damping percentage of $1 \%$ for stable modes and a damping rate of 10 for modes involved in coalescence.

\section{Evaluation of the disc brake stability}

The first step in the stability analysis consists of the calculation of the quasi-static sliding equilibrium configuration $\mathbf{X}_{\text {static }}$ associated with the hydraulic pressure applied. This configuration is defined by the non-linear static problem which corresponds to Equation 3.

$$
\mathrm{KX}_{\text {static }}=\mathbf{F}_{\mathrm{NL}}\left(\mathrm{X}_{\text {static }}\right)+\mathbf{F}_{\max }
$$

The non-linear equations of motion are linearized around the sliding equilibrium point and a complex eigenvalue analysis is performed with respect to the friction coefficient and the braking pressure. Figure 2 shows the stability results for a pressure in the range [1; 100] bars and a friction coefficient in the range $[0 ; 1]$. Figure 2 (a) represents the stability results over the complex plane and points with a positive real part correspond to the unstable sliding equilibriums. Two main areas contain all the unstable modes: between 9420 and $9480 \mathrm{~Hz}$ and between 900 and $1650 \mathrm{~Hz}$. Figure 2 (b) shows the number of unstable modes with respect to the constant pressure $P_{\max }$ applied and the friction coefficient. Five domains are noted and correspond to sliding equilibrium configurations with different stability results: from stable (no unstable mode) up to five unstable modes. This analysis allows for select the couple of parameters $\left(\mu, P_{\max }\right)$ which provides the wanted instability. In this work, the single instability case defined by $\mu=0.72$ and $P_{\max }=12.5$ bars is investigated.

\section{Self-excited vibrations}

The previous set of parameters presents one unstable mode at $930 \mathrm{~Hz}$ but according to Figure 2 (b), it can be noted that the system is close to the two unstable modes domain. In the previous section, the stability analysis does not consider the ramp loading defined by $\mathbf{F}(t)$ but only the loading force vector associated with the maximum pressure (i.e. $\mathbf{F}(t)=\mathbf{F}_{\max }$ ). So, time integration needs to be performed to investigate the effects of the progressive load.

This section aims at comparing the time and acoustic responses of the brake system for two kinds of initial and loading conditions. In the literature, studies are carried out by initializing temporal integration around the quasi-static sliding equilibrium configuration $\mathbf{X}_{\text {static }}$ with a disturbance around this point. In [9], the effects of the initial disturbance on the dynamic response is presented. The author shows that initial condition can significantly change the brake response. The main disadvantages of this method is the fact that the non-linear vibrations during the achievement of the sliding equilibrium are neglected. In this paper, the focus is on the influence of ramp loading with time integration initialized with the configuration without loading.

Cases under study. The two cases under study both present a single instability and correspond to the following parameters: 

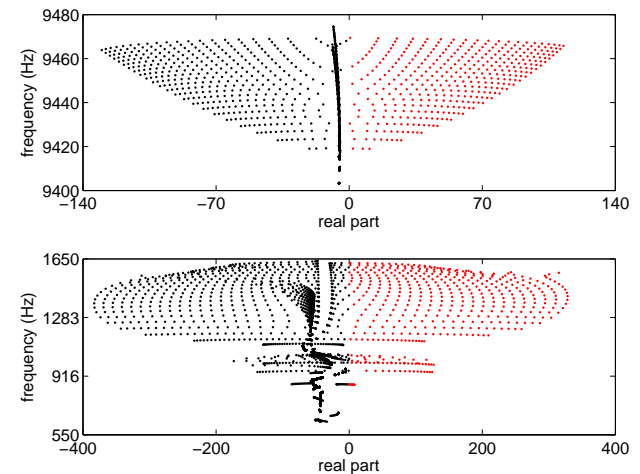

(a)

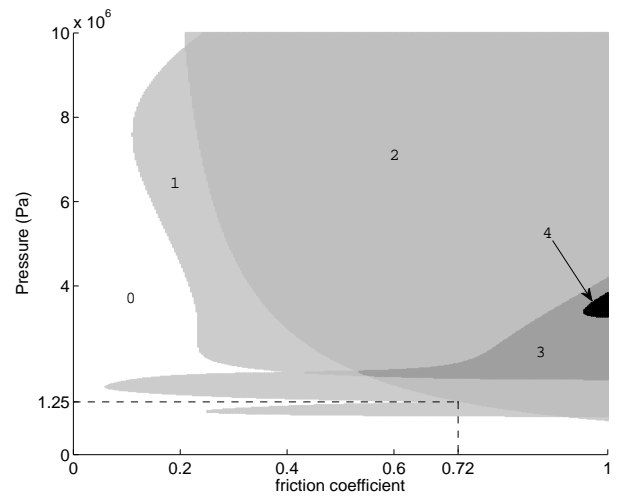

(b)

Fig. 2: Stability analysis calculated for $P_{\max } \in[1 ; 100]$ bars and $\mu \in[0 ; 1]$. (a): real part vs frequency; (b): Areas of stability: from white to black 0 unstable mode (stable case), 1, 2, 3 and 4 unstable modes

- Case 1: $\mu=0.72, \mathbf{F}(t)=\mathbf{F}_{\max }, \mathbf{X}(t=0)=\mathbf{X}_{\text {static }}+\varepsilon, \dot{\mathbf{X}}(t=0)=\mathbf{0}$

- Case 2: $\mu=0.72, \mathbf{F}(t)$ ramp loading, $t_{R}=10^{-3}$ s (see Equation 1), $\mathbf{X}(t=0)=\mathbf{0}, \dot{\mathbf{X}}(t=0)=\mathbf{0}$ where $\varepsilon$ defines a small disturbance around the sliding equilibrium point $\mathbf{X}_{\text {static }}$. It can be noted that no disturbance on the dynamic response of the system is introduced for case 2.

Continuous Wavelet Transform. To compare cases 1 and 2, spectrum analysis are performed by using the Continuous Wavelet Transform method (CWT). The signal processing method provides a good description of the non-linear contributions which occurs during the transient and stationary responses. The method consists of the decomposition of a signal into wavelets that are well localized both in time and frequency. For a time signal $f(t)$, the CWT is defined by Equation 4:

$$
W(a, b)=\int_{-\infty}^{+\infty} f(t) \Psi_{a, b}^{*}(t) d t \quad \text { where } \Psi_{a, b}(t)=\frac{1}{\sqrt{a}} \Psi\left(\frac{t-b}{a}\right)
$$

where $\Psi_{a, b}(t)$ are the daughter wavelets, $a$ defines the scale parameter, and $b$ corresponds to the time parameter. The asterisk $\Psi_{a, b}^{*}$ indicates the complex conjugate of $\Psi_{a, b}$. The daughter wavelets $\Psi_{a, b}(t)$ are given by the kind of mother wavelet that has to be chosen first. The following admissibility condition has to be satisfied $0<C_{\Psi}<+\infty$ where $C_{\Psi}$ defines the admissibility constant $C_{\Psi}=\int_{-\infty}^{+\infty} \frac{\mid \hat{\Psi}(\omega \mid}{|\omega|} d \omega$ and $\hat{\Psi}$ is the Fourier transform of $\Psi$. For a time signal $f(t)$ represented by $N$ sampled, data points (with uniform time step $\delta t$ ), the Continuous Wavelet Transform of Equation (4) is a convolution of the data sequence $f\left(n^{\prime}\right)$ (with $n^{\prime}=1, \ldots, N$ ) with a scaled and normalized wavelet. It can be represented by Equation 5:

$$
C(a, n)=\sum_{n^{\prime}=0}^{N-1} f\left(n^{\prime}\right) \sqrt{\frac{\delta t}{a}} \Psi_{0}^{*}\left(\frac{\left(n^{\prime}-n\right) \delta t}{a}\right)
$$

where $n$ defines the localized time index and $\delta t$ is the sampling interval. The most important point is the specification of an appropriate type of mother wavelet to provide the previously defined daughter wavelets. In reality, the mother wavelet is scaled and shifted to build basis functions which are the daughter wavelets. In this study, the Morlet mother wavelet has been chosen due to the fact that it is one of the most commonly used CWT wavelets (and it is well localized in both the time and frequency domain). It is defined in the time domain by: $\Psi_{0}(\eta)=\pi^{-\frac{1}{4}} e^{i m \eta} e^{-\frac{\eta^{2}}{2}}$ where $m$ is the wave number and $\eta$ is a non-dimensional time parameter. The wavelet function contains unit energy at every scale due to the normalization of the mother wavelet. The wavelet power is then defined as $|C(a, n)|^{2}$. A 
complete theoretical background can be found in [10].

Time responses. Figure 3 (a) presents the velocity calculated for case 1 for a normal dof of the friction interface. This response presents the classical features of self-excited vibrations with a single instability and similar responses can be found in $[9,4]$. Actually, the amplitude of velocity increases during a transient regime until it reaches a stationary regime. A spectrum analysis is performed by using the CWT previously defined. Figure 3 (b) shows the wavelet power spectrum for case 1 . It can be noted that the frequency $f_{1}$ and its harmonic components such as $2 f_{1}$ are present but the component $f_{1}$ is predominant. The fundamental frequency $f_{1}$ is predicted by the stability analysis and appearance of its harmonic is due to non-linearities.

Figure 3 (c) illustrates the time response for case 2 and it can be noted that it is significantly different from the case 1. Actually, the profile of the velocity exhibits a short transient regime where the amplitude is much greater than stationary (for $t \in[0.1 ; 0.2] \mathrm{s}$ ). By comparing the velocities of cases 1 and 2, it can be noted that the amplitude for case 2 is about 130 times higher than for case 1 . Moreover, the wavelet power spectrum associated with case 2 shows a spectrum with more harmonic components than for the case 1 (see Figure $3(\mathrm{~d})$ ). The two frequencies $f_{1}=944 \mathrm{~Hz}$ and $f_{2}=9424$ $\mathrm{Hz}$ and several harmonic combinations appears in the spectrum. The first frequency $f_{1}$ is not predicted by the stability analysis but it is close to the prediction of $930 \mathrm{~Hz}$ : there is an evolution of $f_{1}$ due to the evolution of the quasi-static sliding equilibrium and non-linearities. Actually, the comparison between the sliding equilibrium and temporal evolution of the average of the displacement shows an important gap of about $40 \%$ : the system does not oscillate around its sliding equilibirum. In case 1, this gap does not exist, the brake system position is near the sliding equilibrium throughout the response. In case 2 , the wavelet power spectrum is composed of the frequency $f_{1}$ and its harmonic components $2 f_{1}, 3 f_{1}$, $4 f_{1}$ etc. The second frequency $f_{2}$ is present at the begining of the response but disappears for $t \geq 1.5$ $\mathrm{s}$ and this is sufficient to activate the component $-10 f_{1}+f_{2}$ throughout the response. However, it can be seen that the components $f_{1}$ and $2 f_{1}$ are predominant and lead the response. Involving the component $f_{2}$, it is not predicted by the stability associated with the current set of parameters $(\mu=0.72$, $P_{\max }=12.5$ bars) but with another wich provides two unstable modes (for example $\mu=0.74$ and $P_{\max }=12.5$ bars).

To conclude, case 1 provides a response led by $f_{1}=930 \mathrm{~Hz}$, whereas case 2 provides a response where $f_{1}=944 \mathrm{~Hz}$ and $2 f_{1}$ are predominant. The frequency $f_{2}=9424 \mathrm{~Hz}$ appears in case 2 and activates harmonic components of the form $\pm m f_{2} \pm n f_{2}$ (where $n$ and $m$ are positive integers) which are not predominant. So, the case 1 is a single instability case with low amplitude whereas case 2 seems to be a multi-instability case with high amplitude. It can be noted that stability analysis does not predict all the unstable modes that govern the dynamic response for case 2 and the brake system can jump into another area of stability for a sufficiently fast progressive load.

Acoustic radiation. In this paragraph, sound pressure radiated during squeal event is characterized in terms of level and directivity. The method applied to perform this calculation is based on the three following steps. Firstly, non-linear time integrations are performed. Secondly, a Fourier transform is applied in order to build a Fourier basis which contains all the harmonic components present in the response and the field of velocity is decomposed by order. For the following calculations, all the orders previously found in the non-linear response are retained. Thirdly, the boundary element method is applied for each order due to the fact that the pressure needs to be calculated for a unique frequency. Then the global sound pressure is obtained by superposition. The level of acoustic intensity is defined by the following expression: $L_{d B}=10 \log _{10}\left(\mathbf{P P}^{*} / P_{r e f}^{2}\right)$, where $\mathbf{P}$ denotes the calculated sound pressure and $P_{r e f}$ is the minimum audible sound pressure $\left(P_{r e f}=2 \times 10^{-5} \mathrm{~Pa}\right)$. The estimation of $L_{d B}$ in the far and near fields is performed over two observation planes placed at $5 \times 10^{-2}$ and 1 meter high from the top of the pad. The directivity pattern is evaluated over four planes defined by $P_{0}, P_{\frac{\pi}{4}}, P_{\frac{\pi}{2}}$ and $P_{\frac{3 \pi}{4}}$ (see Figure 1 (c)).

Figure 4 shows the acoustic intensity $L_{d B}$ corresponding to the case 1 . Over the mesh (Figure 4 (a)), $L_{d B}$ presents variations from 100 to $124 \mathrm{~dB}$ over the pad, 93 to $115 \mathrm{~dB}$ over the disc and the 


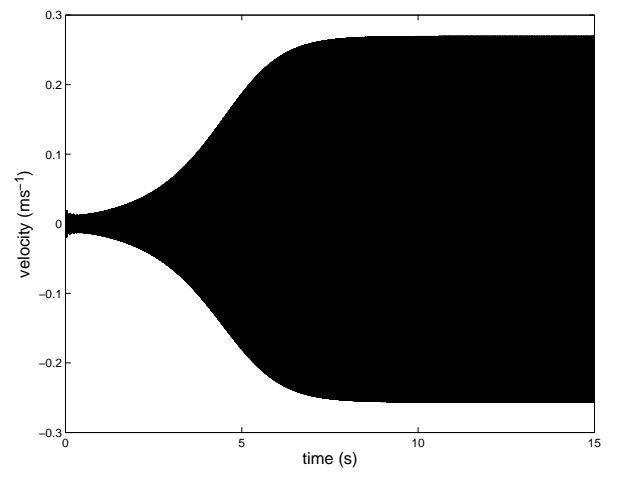

(a)

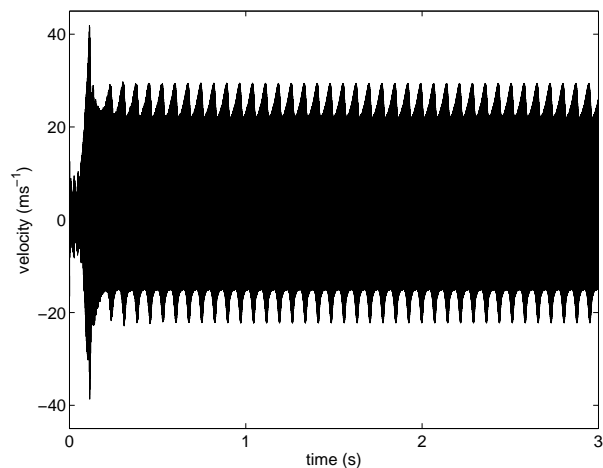

(c)

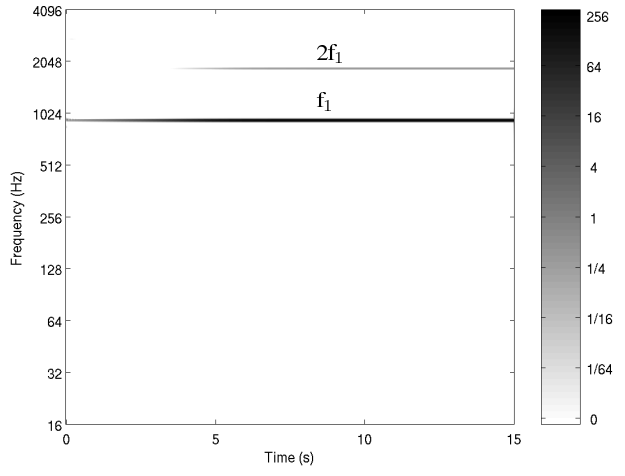

(b)

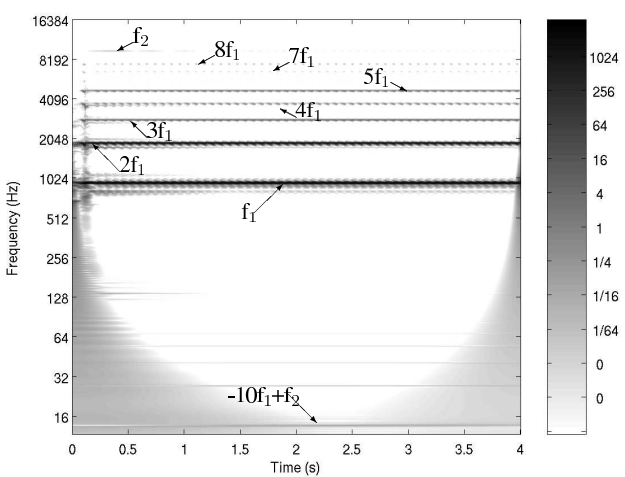

(d)

Fig. 3: Time responses and wavelet power spectrum associated with a normal dof of the interface. (a): velocity in case 1; (b): wavelet power spectrum in case 1; (c): velocity in case 2 (d): wavelet power spectrum in case 2

maximum is reached over the pad. In the near field (Figure 4 (b)), a peculiar intensity pattern can be noted, four directivity lobes clearly appear and the maximum level is about $127 \mathrm{~dB}$. By comparing Figures 4 (a) and (b), it can be seen that the intensity pattern in the near field corresponds to the pattern over the mesh: the four lobes present in the near field correspond to the four areas of maximum intensity over the mesh. In the far field (Figure 4 (c)), the profile of propagation is mainly composed of two lobes: the lobes in the near field disappear. Moreover, it can be noted that the maximum is about $82 \mathrm{~dB}$. The three previous Figures also show that levels of sound pressure decrease with the distance from the source. Figures 4 (d), (e), (f) and (g) show $L_{d B}$ over $P_{\theta}$. Only profiles of propagation are presented, the levels are not displayed and the color map has been chosen to emphasize the wave front lines. It can be seen that the patterns of directivity over $P_{0}$ and $P_{\pi / 4}$ (Figures 4 (d) and (e)) are similar, both present a central lobe and the propagation is mainly along $\mathrm{z}$-axis: sound pressure is unidirectional. Over $P_{3 \pi / 4}$ and $P_{\pi / 2}$, it can be noted that the propagation is omnidirectional as illustrated in Figures 4 (f) and (g). In [11], the author proposes a numerical and experimental study of brake squeal which presents a single instability (as for the case 1). Temporal integration is performed with the same conditions as for the case 1 . The numerical results are similar with those of case 1: the amplitude of velocity are close and the spectrums are composed of $f_{1}$ and its harmonic. The results of the case 1 are in accordance with the acoustic response in [11] that was estimated experimentally with a similar disc brake system. Another relevant work is presented in [12]: the authors numerically investigate the influence of geometrical and interface meshing parameters over the squeal noise. It is important to note that radiations are calculated by extracting velocity from a forced response and so, does not match the assumptions of the present study. However, the patterns of directivity presented in 


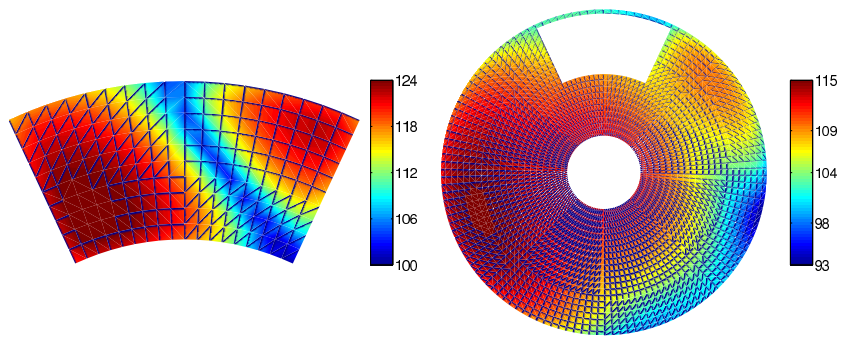

(a)

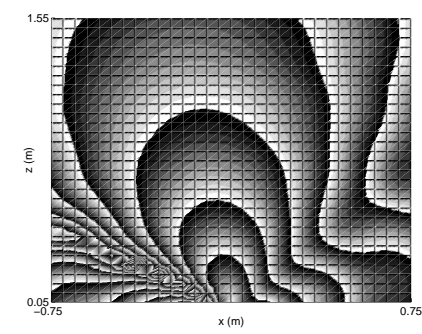

(d)

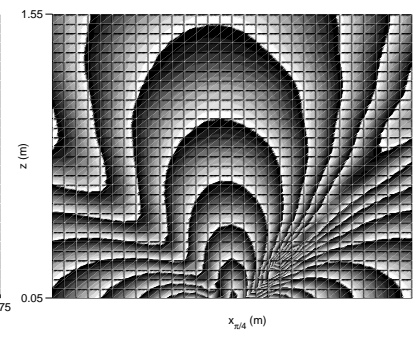

(e)

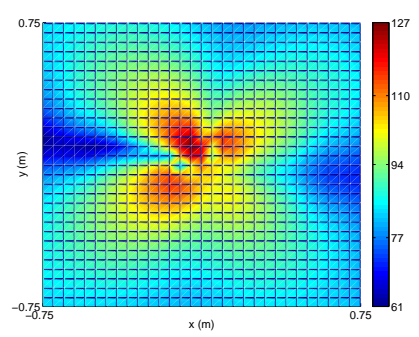

(b)

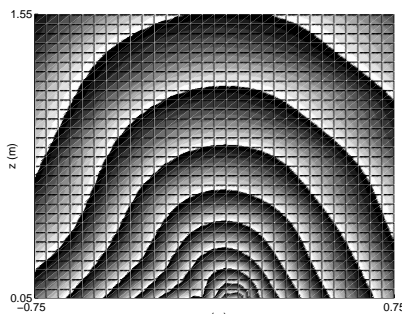

(f)

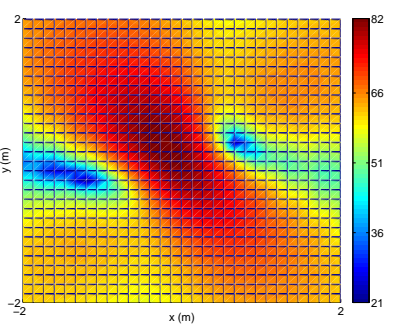

(c)

Fig. 4: $L_{d B}$ for case 1. (a): boundary element mesh; (b): near field; (c): far field; (d): $P_{0}$; (e): $P_{\frac{\pi}{4}}$; (f): $P_{\frac{\pi}{2}} ;(\mathrm{g}): P_{\frac{3 \pi}{4}}$

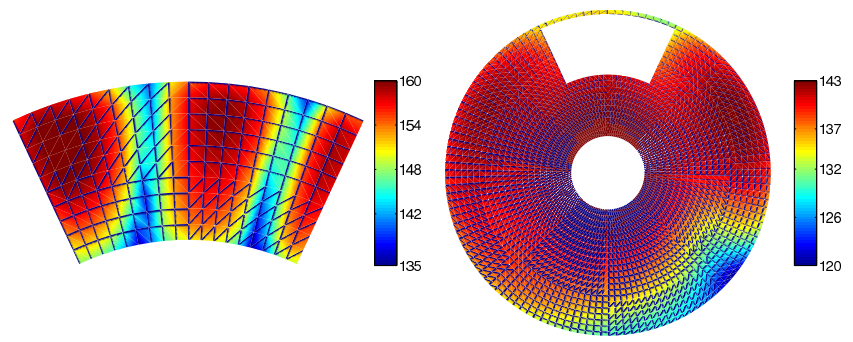

(a)

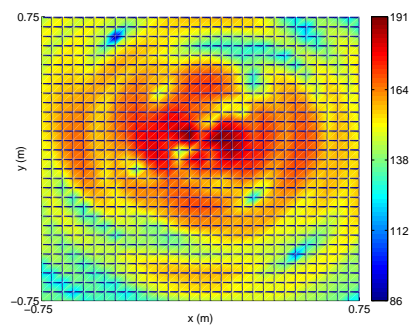

(b)

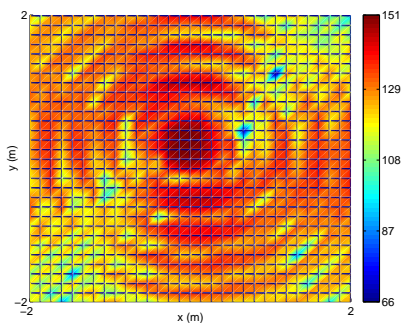

(c)

Fig. 5: $L_{d B}$ for case 2 during the final stationary regime. (a): boundary element mesh; (b): near field; (c): far field

[12] are composed of several lobes and this is similar with the results of the case 1.

For the case 2, acoustic intensity $L_{d B}$ is presented in Figure 5. Over the mesh (Figure 5 (a)) the acoustic intensity differs from case 1: levels are from 135 to $160 \mathrm{~dB}$ over the pad, from 120 to $143 \mathrm{~dB}$ over the disc and the maximum is reached over the pad. The maximum level in the near field is about $191 \mathrm{~dB}$ in contrast to the of $127 \mathrm{~dB}$ for case 1 . This is due to the fact that the amplitude of velocity for case 2 is higher than for case 1. Involving the directivity in the near and far fields, Figures 5 (b) and (c) show peculiar patterns which are mainly composed of circular wave front lines generated at the disc brake center. The directivity of case 2 is different from case 1 due to the different predominant harmonic components which compose the spectrum (i.e. $f_{1}$ for case $1, f_{1}$ and $2 f_{1}$ for case 2 ).

It can be concluded that a progressive load can significantly increase levels of noise emission and modify the pattern of directivity.

\section{Conclusion}

In this paper a simplified model of a brake system is investigated. Classical formulations of the non-linear cubic contact law and friction Coulomb law are used to model the friction interface. The stability analysis associated with this system provides classical cases of instabilities (single and multi- 
instabilities) and an estimation of the areas of stability is performed with respect to the hydraulic pressure and the friction coefficient. The dynamic response is calculated for two cases: case 1 with a static loading and case 2 with a ramp loading. These two cases present different velocities and spectrum analysis shows the appearance of an unexpected second fundamental frequency $f_{2}$ for case 2 . The appearance of this second fundamental frequency is due to both non-linearities and the ramp loading. This kind of loading condition is able to activate new harmonic components which correspond to the classical multi-instability case. It can be concluded that a "sufficiently" fast ramp loading can contradict the stability analysis about the prediction of the fundamental unstable frequencies. For the two previous cases, the sound pressure radiated during stationary regime is calculated. It appears that the levels $L_{d B}$ are significantly higher for case 2 than case 1 and this is due to the difference between the amplitudes of velocity. The directivity patterns for both cases are significantly different: for case 1 , four and two lobes are present in near and far field whereas for case 2 circular waves can be seen.

\section{Acknowledgments}

The first author gratefully acknowledges the French Education Ministry which supports this research. The authors thank the financial support provided by the French National Research Agency through the framework of its project ANR-12-JS09-0009.

\section{References}

[1] H. Ouyang, N. Nack, Y. Yuan, and F. Chen. Numerical analysis of automotive disc brake squeal: a review. International Journal of Vehicule Noise and Vibration, Vol. 1(3/4):207-231, 2005.

[2] N.M. Kinkaid, O.M. O'Reilly, and P. Papadopoulos. Automotive disc brake squeal. Journal of Sound and Vibration, Vol. 267(1):105-166, 2003.

[3] R.A. Ibrahim. Friction-induced vibration, chatter, squeal, and chaos part 1: Mechanics of contact and friction. American Society of Mechanical Engineers, Vol. 47(7):209-226, 1994.

[4] F. Chevillot, J.-J. Sinou, and N. Hardouin. Nonlinear transient vibrations and coexistences of multi-instabilities induced by friction in an aircraft braking system. Journal of Sound and Vibration, Vol. 328(4-5):555-574, 2009.

[5] F. Massi, L. Baillet, O. Giannini, and A. Sestieri. Brake squeal: Linear and nonlinear numerical approaches. Mechanical Systems and Signal Processing, Vol. 21(6):2374-2393, 2007.

[6] V. Cutanda Henriquez and P. Juhl. OpenBEM. http://www.openbem.dk/.

[7] Marc Bonnet. Boundary Integral Equation Methods for Solids and Fluids. John Wiley \& Sons Ltd, 1999.

[8] N. Coudeyras, J.-J. Sinou, and S. Nacivet. A new treatment for predicting the self-excited vibrations of nonlinear systems with frictional interfaces: The constrained harmonic balance method, with application to disc brake squeal. Journal of Sound and Vibration, Vol. 319(3-5):1175-1199, 2009.

[9] J.-J. Sinou. Transient non-linear dynamic analysis of automotive disc brake squeal - on the need to consider both stability and non-linear analysis. Mechanics Research Communications, Vol. 37(1):96-105, 2010.

[10] S. Mallat. Theory of multiresolution signal decomposition: the wavelet representation. Transaction on Pattern Analysis and Machine Intelligence, Vol. 11:674-693, 1989.

[11] U. von Wagner, T. Jearsiripongkul, T. Vomstein, G. Chakraborty, and P. Hagedorn. Brake squeal: Modeling and experiments. VDI-Bericht, Vol. 1749:96-105, 2010.

[12] S. Oberst, J. C. S. Lai, and S. Marburg. Guidelines for numerical vibration and acoustic analysis of disc brake squeal using simple models of brake systems. Journal of Sound and Vibration, Vol. 332(9):2284-2299, 2013. 